\title{
DESIDRATAÇÃO DE ETANOL SOBRE MATERIAL NANOESTRUTURADO DO TIPO LaSBA-15
}

\author{
Geraldo E. Luz Jr.* \\ Coordenação de Química, Centro de Ciências da Natureza, Universidade Estadual do Piauí, Campus Poeta Torquato Neto, $64002-150$ \\ Teresina - PI, Brasil \\ Ana C. R. Melo, Stevie H. Lima, Antônio S. Araujo e Valter J. Fernandes Jr. \\ Departamento de Química, Universidade Federal do Rio Grande do Norte, 59072-970 Natal - RN, Brasil
}

Recebido em 6/10/09; aceito em 19/4/10; publicado na web em 20/7/10

\begin{abstract}
ETHANOL DEHYDRATION OVER LaSBA-15 NANOSTRUTURED MATERIAL. La-incorporated SBA-15 mesopourous molecular sieves (LaSBA-15) were directly synthesized with aim to convert ethanol to ethylene. The samples were characterized by XRD, XRF, nitrogen sorption and acidity, by thermodesorption of n-buthylamine. The results have indicated that all the samples have showed high ordered mesostructure with a large average pore size, and that the lanthanum incorporation has caused an increase in the acidity of the SBA-15. The LaSBA-15 samples have improved, with low deactivation rate, the conversion of the ethanol to water, ether, acetaldehyde and ethylene. In addition, they have increased the ethylene selectivity.
\end{abstract}

Keywords: ethanol dehydration; LaSBA-15; ethylene.

\section{INTRODUÇÃO}

A conversão catalítica de alcoóis em hidrocarbonetos e em outros compostos oxigenados, como éteres, foi impulsionada na década de 1970 pela crise do petróleo. Atualmente, a crescente preocupação com as questões ambientais é a principal motivação para pesquisas nesta área. Desde a citada década, vários catalisadores foram estudados, como alumina ${ }^{1}$ e a zeólita ZSM-5; ${ }^{2}$ indicando que tal desidratação pode ocorrer sobre sítios ácidos moderados e que a mesma pode ser intramolecular ou intermolecular. ${ }^{1}$ A primeira, leva à formação de olefinas; ${ }^{2,3}$ enquanto a segunda, promove a formação de éter. ${ }^{3}$ Além disso, o álcool pode sofre desidrogenação sobre sítios básicos com formação de aldeído ou cetona. ${ }^{4}$ Nos últimos anos, peneiras moleculares mesoporosas com excelentes propriedades texturais têm sido utilizadas no desenvolvimento de sistemas catalíticos com sítios ativos bem dispersos. ${ }^{5}$ Estes materiais mesoporosos têm a vantagem de apresentarem uma baixa taxa de coqueamento em decorrência do elevado diâmetro médio de poros. Dentre estas peneiras moleculares, destaca-se a SBA-15, material nanoestruturado com mesoporos unidirecionais organizados de forma hexagonal e interligados por microporos, e que apresenta elevada área superficial e diâmetro médio de poros no intervalo de $2-30 \mathrm{~nm} .{ }^{6}$ No entanto, este material não apresenta sítios ácidos na forma de sílica pura. Por conta disto, é comum a impregnação de metais, como as terras raras, ${ }^{7,8}$ como meio de conferir acidez a esta peneira molecular; embora tal processo não seja fácil em virtude da elevada solubilidade dos íons metálicos no gel de síntese $(\mathrm{pH}<1) .{ }^{8}$ Baseado nestas informações, este trabalho teve como objetivo avaliar a ação catalítica de materiais nanoestruturados do tipo LaSBA-15 na desidratação de etanol.

\section{PARTE EXPERIMENTAL}

\section{Síntese e caracterização dos catalisadores}

As amostras de LaSBA-15, com diferentes razões molares Si/La (75, 50 e 25), foram sintetizadas pelo método hidrotérmico com ajuste do $\mathrm{pH}$ do gel de síntese a 6, após 24 h de tratamento hidrotérmico,

\footnotetext{
*e-mail: geraldoeduardo@gmail.com
}

como proposto por Luz Jr. et al.. ${ }^{9}$ Para isto, foram utilizados o copolímero tribloco Pluronic P123 $\left(\mathrm{EO}_{20} \mathrm{PO}_{70} \mathrm{EO}_{20}\right)$ da BASF Co., ácido clorídrico da Vetec, $37 \%$, água destilada, tetraetilortossilicato (TEOS) da Aldrich, como fonte de sílica, e cloreto de lantânio hepta-hidratado $\left(\mathrm{LaCl}_{3} \cdot 7 \mathrm{H}_{2} \mathrm{O}\right)$ da AnalaR (BDH), a 99\%, como fonte de lantânio. Em todas as amostras foi utilizada a razão molar 1,000 TEOS: 0,015 P123: $2,750 \mathrm{HCl}: 166,0 \mathrm{H}_{2} \mathrm{O}$ e a quantidade de $\mathrm{LaCl}_{3} .7 \mathrm{H}_{2} \mathrm{O}$ necessária para obtenção de cada uma das razões molares $\mathrm{Si} / \mathrm{La}$ citadas anteriormente. Inicialmente, o P123 foi misturado à solução aquosa de $\mathrm{HCl}$, ficando sob agitação por $3 \mathrm{~h}$ a $40^{\circ} \mathrm{C}$. Em seguida, adicionaram-se o TEOS e o $\mathrm{LaCl}_{3} .7 \mathrm{H}_{2} \mathrm{O}$. A mistura resultante ficou sob agitação por $24 \mathrm{~h}$ a $40{ }^{\circ} \mathrm{C}$. $\mathrm{O}$ gel resultante foi transferido para um recipiente de teflon, onde foi mantido em repouso a $100^{\circ} \mathrm{C}$ por $24 \mathrm{~h}$. Depois desse tempo, o recipiente foi resfriado à temperatura ambiente, quando, então, o gel teve seu $\mathrm{pH}$ ajustado a 6,0 com n-butilamina anidra com o objetivo de facilitar a impregnação do metal, como reportado por Luz Jr et al.. ${ }^{9}$ Em seguida, foi novamente aquecido a $100{ }^{\circ} \mathrm{C}$ e mantido nesta temperatura por mais $24 \mathrm{~h}$, em repouso. O sólido obtido foi filtrado, à temperatura ambiente, lavado com etanol anidro, seco por $48 \mathrm{~h}$ e, finalmente, calcinado a 550 ${ }^{\circ} \mathrm{C}$ sob atmosfera dinâmica de nitrogênio $\left(100 \mathrm{~mL} \mathrm{~min}^{-1}, 10{ }^{\circ} \mathrm{C} \mathrm{min}{ }^{-1}\right)$ por $2 \mathrm{~h}$ e, por mais $2 \mathrm{~h}$, à mesma temperatura, sob atmosfera de ar (100 $\left.\mathrm{mL} \mathrm{min}^{-1}\right) .{ }^{10}$ As amostras, assim sintetizadas, foram denominadas de La $\mathrm{SBA}-15$, onde " $\mathrm{x}$ " indica a razão molar Si/La no gel de síntese. Utilizando procedimento semelhante, foi sintetizada uma amostra de SBA-15 sem lantânio, que foi denominada SiSBA-15. Todas as amostras foram caracterizadas por difratometria de raios-X, fluorescência de raios-X, adsorção/dessorção de nitrogênio e acidez total, pelo método de termodessorção de n-butilamina. ${ }^{11}$

\section{Desidratação de etanol}

Os ensaios de desidratação de etanol foram realizados em uma unidade de avaliação catalítica, modelo TCAT-10, acoplado a um cromatógrafo a gás Varian CP3800, equipado com uma coluna de polidimetilsiloxano $(60 \mathrm{~m}, 0,53 \mathrm{~mm}, 5 \mu \mathrm{m})$ e detector de condutividade térmica. Os testes foram realizados com $60 \mathrm{mg}$ de amostra sob fluxo de $\mathrm{H}_{2}$ de $37 \mathrm{~mL} \mathrm{~min}^{-1}$, a $500{ }^{\circ} \mathrm{C}$. Antes de cada ensaio, os catalisadores foram ativados por $2 \mathrm{~h}$ sob as mesmas condições de temperatura e fluxo dos testes catalíticos. A identificação dos produtos foi realizada 
através da comparação dos tempos de retenção dos picos de cada cromatograma com os tempos de retenção de padrões de etanol, água, éter dietílico, eteno e acetaldeído. Os testes foram conduzidos com todos os catalisadores na forma de pós, com o objetivo de minimizar os efeitos provenientes do transporte interno de massa.

\section{RESULTADOS E DISCUSSÃO}

\section{Caracterização dos catalisadores}

As razões molares reais $\mathrm{Si} / \mathrm{La}$ obtidas por fluorescência de raios-X, apresentadas na Tabela 1 , indicam que o método de síntese com ajuste de $\mathrm{pH}$ possibilitou a incorporação de uma considerável quantidade de lantânio na peneira molecular do tipo SBA-15, especialmente na amostra $\mathrm{La}_{25} \mathrm{SBA}-15$. Além disso, observa-se que o aumento da concentração de lantânio no gel de síntese favoreceu a incorporação do mesmo.

Tabela 1. Razão molar Si/La nas amostras sólidas LaSBA-15

\begin{tabular}{lcc}
\hline Amostra & Si/La no gel & Si/La no sólido \\
\hline $\mathrm{La}_{75} \mathrm{SBA}-15$ & 75 & 319,6 \\
$\mathrm{La}_{50} \mathrm{SBA}-15$ & 50 & 143,9 \\
$\mathrm{La}_{25} \mathrm{SBA}-15$ & 25 & 44,6 \\
\hline
\end{tabular}

Os difratogramas de raios-X de todas as amostras estão apresentados na Figura 1 e demonstram que todas elas apresentam três picos de difração referentes aos planos de difração (100), (110) e (200), característicos da estrutura mesoporosa bidimensional com simetria P6mm, como aquela presente em materiais do tipo SBA-15. Além disso, houve um aumento da intensidade dos citados picos nas amostras LaSBA-15 em relação à amostra SiSBA-15, indicando que a incorporação do lantânio provocou um aumento da cristalinidade da peneira molecular em questão. Também foi observado o deslocamento do pico referente ao plano de difração (100) para valores menores de $2 \theta$, à medida que a concentração de lantânio nas amostras sólidas aumentou. Tal deslocamento é decorrente do aumento do parâmetro de rede, e pode indicar um aumento do diâmetro médio de poros ou da espessura da parede dos poros.

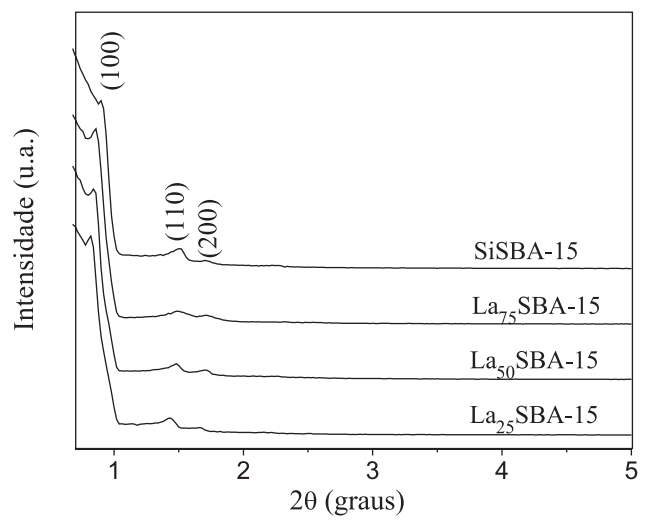

Figura 1. Difratogramas de raios-X das amostras SiSBA-15 e LaSBA-15

As isotermas de adsorção/dessorção de $\mathrm{N}_{2}$, apresentadas na Figura 2, indicam que todas as amostras apresentaram isotermas do tipo IV com histerese do tipo I, características de materiais mesoporosos com sistema cilíndrico de poros, como a SBA- $15 .{ }^{6}$

Os valores da área superficial, diâmetro médio de poros e volume poroso, obtidos a partir das isotermas apresentadas na Figura 2, estão apresentados na Tabela 2, juntamente com os dados do parâmetro de

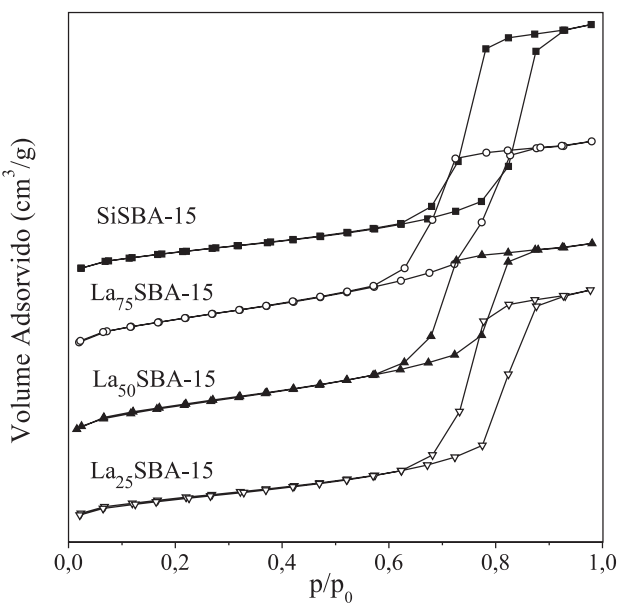

Figura 2. Isotermas de adsorção/dessorção de $N_{2}$ das amostras SiSBA-15 e LaSBA-15

rede $\left(a_{0}\right)$ e da espessura da parede dos poros $(w)$. Os dados demonstraram que a incorporação do lantânio à SBA-15 provocou aumento do parâmetro de rede, como sugerido pelos difratogramas de raios-X, e da espessura da parede. Além disso, causou aumento da área superficial e diminuição do diâmetro médio de poros e do volume poroso. Tais alterações foram mais intensas à medida que a concentração de lantânio na peneira molecular diminuiu, podendo estar relacionadas à presença de uma fina camada de óxido de lantânio no interior dos mesoporos da peneira molecular, a qual pode bloquear parcialmente os poros, aumentando a espessura da parede, além de aumentar a área superficial da peneira molecular, por elevar a rugosidade superficial. A forma como a intensidade das referidas alterações aumenta, associada ao comportamento da espessura da parede, indica que a camada de óxido de lantânio está mais presente na amostra $\mathrm{La}_{75} \mathrm{SBA}-15$ (menor teor de lantânio) do que na $\mathrm{La}_{25}$ SBA-15 (maior teor de lantânio). A presença desta camada de óxido também pode ser a explicação para a maior acidez total das amostras de LaSBA-15 em relação à amostra SiSBA-15, conforme os dados apresentados na Tabela 3. Os dados desta tabela também demonstram que o aumento da acidez total das amostras LaSBA-15 é coerente com o aumento da presença desta camada de óxido de lantânio.

Tabela 2. Propriedades texturais das amostras SiSBA-15 e LaSBA-15

\begin{tabular}{lccccc}
\hline Amostra & $\mathrm{S}_{\text {BET }}\left(\mathrm{m}^{2} / \mathrm{g}\right)$ & $\mathrm{Vp}\left(\mathrm{cm}^{3} / \mathrm{g}\right)$ & $\mathrm{Dp}(\mathrm{nm})$ & $a_{0}{ }^{\mathrm{a}}(\mathrm{nm})$ & $w^{\mathrm{b}}(\mathrm{nm})$ \\
\hline SiSBA-15 & 589,8 & 1,46 & 7,21 & 11,33 & 4,12 \\
$\mathrm{La}_{75} \mathrm{SBA}-15$ & 775,4 & 1,31 & 4,27 & 11,86 & 7,59 \\
$\mathrm{La}_{50} \mathrm{SBA}-15$ & 720,2 & 1,18 & 5,35 & 12,14 & 6,79 \\
$\mathrm{La}_{25} \mathrm{SBA}-15$ & 557,5 & 1,49 & 5,37 & 12,44 & 7,07
\end{tabular}

${ }^{\mathrm{a}} a_{0}=2 \mathrm{~d}_{(100)} / \sqrt{ } 3 ;{ }^{\mathrm{b}} w=a_{0}-\mathrm{Dp}$

Tabela 3. Acidez total das amostras SiSBA-15 e LaSBA-15

\begin{tabular}{lc}
\hline Amostra & Acidez (mmol n-but/g) \\
\hline SiSBA-15 & 1,80 \\
$\mathrm{La}_{75} \mathrm{SBA}-15$ & 3,49 \\
$\mathrm{La}_{50} \mathrm{SBA}-15$ & 2,92 \\
$\mathrm{La}_{25} \mathrm{SBA}-15$ & 2,09 \\
\hline
\end{tabular}

\section{Desidratação de etanol}

Os ensaios de desidratação de etanol demonstraram que as amostras de LaSBA-15 foram capazes de converter o etanol a água, etileno, éter dietílico e acetaldeído. Além disso, a Figura 3, que apresenta a 
conversão do etanol sobre cada uma das amostras ao longo do tempo, demonstra que as amostras com lantânio incorporado apresentaram uma conversão bem maior que a observada sobre a amostra SiSBA-15, indicando que sítios ácidos gerados pela incorporação do lantânio aumentaram a atividade catalítica da peneira molecular em questão. Outro fato observado na Figura 3 é a baixa perda de conversão ao longo do tempo sobre as amostras LaSBA-15, o que indica uma baixa taxa de coqueamento destas amostras. Tal comportamento pode estar relacionado à ausência de produtos de polimerização, evidência da moderada acidez das amostras catalíticas, e ao elevado diâmetro médio de poros destas amostras, que permite uma boa fluidez dos produtos.

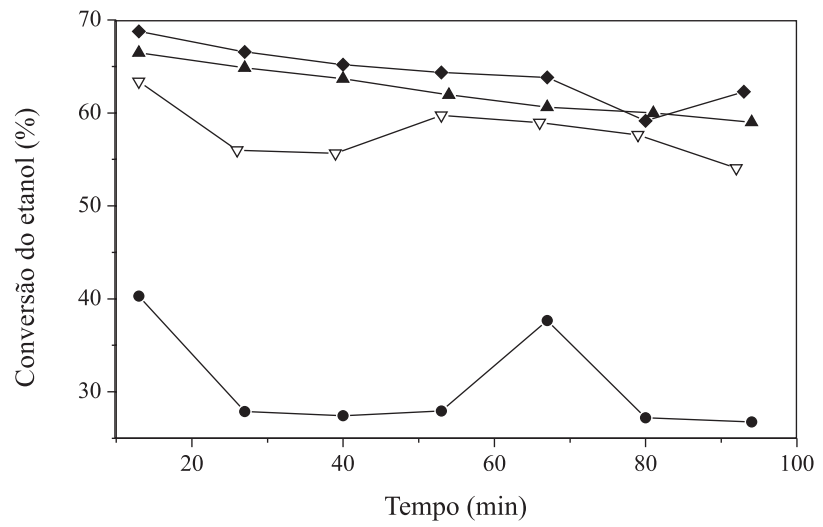

Figura 3. Conversão do etanol sobre as amostras SiSBA-15 (-•-), La ${ }_{75} S B A-15$

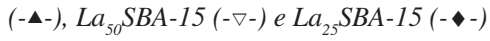

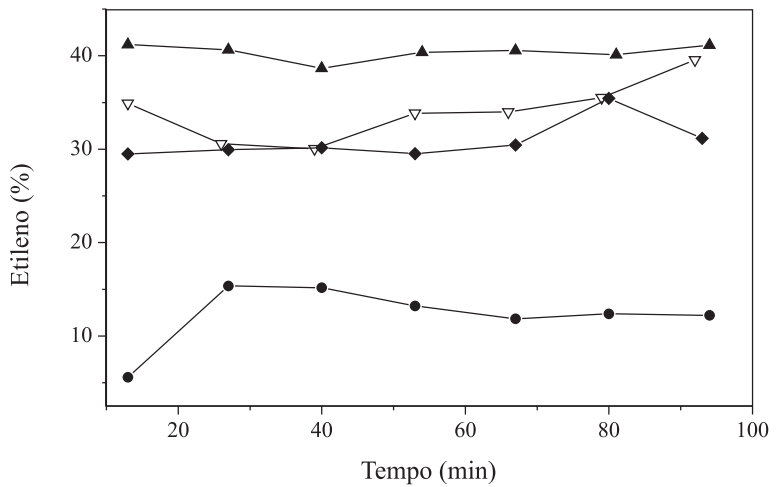

Figura 4. Seletividade para etileno sobre as amostras SiSBA-15 (-•-), $L a_{75} S$ $B A-15$ (-^-), $L a_{50} S B A-15$ (- -) e $L a_{25} S B A-15$ (- -)

Em relação à seletividade, todas as amostras impregnadas com lantânio mostraram-se mais seletivas para etileno. Sendo esta seletividade praticamente constante ao longo do tempo e crescente com a diminuição do teor de lantânio impregnado nas amostras, como apresentado na Figura 4. Este comportamento reflete o comportamento da acidez total das amostras, mencionado anteriormente. Houve uma baixa seletividade para éter dietílico sobre todas as amostras, que pode estar relacionada à elevada temperatura reacional, que não é favorável à desidratação intermolecular (fenômeno exotérmico). A amostra SiSBA-15 mostrou-se mais seletiva para acetaldeído, indicando o predomínio da desidrogenação do etanol sobre os grupos $\mathrm{Si}-\mathrm{O}-\mathrm{Si}$ desta amostra. Este produto também foi obtido, embora em baixas concentrações, nos testes realizados sobre as amostras LaSBA-15, o que indica o caráter anfótero destas, já que a desidrogenação de álcool é um processo reconhecidamente catalisado por base. ${ }^{4}$ Neste caso, os átomos de oxigênio adjacentes a um átomo de lantânio, na fina camada do óxido deste metal, podem ter atuado como sítios básicos.

\section{CONCLUSÃO}

Os resultados de caracterização das amostras de LaSBA-15 e SBA-15 demonstraram que o ajuste de $\mathrm{pH}$ do gel de síntese a 6 com n-butilamina possibilitou a incorporação de lantânio à peneira molecular em questão sob a foram de óxido de lantânio e que tal incorporação proporcionou um aumento de cristalinidade, acidez e da área superficial das amostras. Estas alterações, especialmente o aumento da acidez, proporcionaram uma boa conversão do etanol sobre as amostras LaSBA-15, sendo estas bem seletivas para etileno. Além disso, o elevado diâmetro médio de poros, aliado à acidez moderada das amostras, proporcionou uma baixa taxa de coqueamento e a não diminuição da seletividade para etileno ao longo do tempo.

\section{MATERIAL SUPLEMENTAR}

Neste material, disponível em http://quimicanova.sbq.org.br, na forma de arquivo .PDF, com acesso livre, há uma ilustração da estrutura de poros da peneira molecular do tipo SBA-15.

\section{AGRADECIMENTOS}

Ao CNPq, à CAPES, FAPEPI, UESPI, Petrobras e ANP.

\section{REFERÊNCIAS}

1. Ciola, R.; Fundamentos de Catálise, Moderna: São Paulo, 1981.

2. Machado, N. R. C. F.; Calsavara, V.; Astrath, N. G. C.; Matsuda, C. K.; Paesano Jr., A.; Fuel 2005, 84, 2064; Lima, S. H.; Silva, A. O. S.; Souza, M. J. H.; Araujo, A. S.; Fernandes Jr., V. J.; Carvalho, J. M.; Silva, A. J. N.; Resumos do $3^{\circ}$ Congresso Brasileiro de P\&D em Petróleo e Gás, Salvador, Brasil, 2005; Machado, N. R. C. F.; Calsavara, V.; Astrath, N. G. C.; Medina Neto, A.; Baesso, M. L.; Appl. Catal., A 2006, 311, 193; Calsavara, V.; Baesso, M. L.; Machado, N. R. C. F.; Fuel 2008, 87, 1628; Ramesh, K.; Hui, L. M.; Han, Y.; Borgna, A.; Catal. Commun. 2009, 10, 567.

3. Varisli, D.; Dogu, T.; Dogu, G.; Chem. Eng. Sci. 2007, 62, 5349.

4. Chimentão, R.; Herrera, J. E.; Kwak, J. H.; Medina, F.; Wang, Y.; Peden, C. H. F.; Appl. Catal., A 2007, 332, 263.

5. Mu, Z.; Li, J. J.; Hao, Z. P.; Qiao, S. Z.; Microporous Mesoporous Mater. 2008, 113, 72; Selvaraj, M.; Kawi, S.; Catal. Today 2008, 131, 82; Aguado, J.; Calleja, G.; Carrero, A.; Moreno, J.; Chem. Eng. J. 2008, $137,443$.

6. Zhao, D.; Feng, J.; Huo, Q.; Melosh, N.; Fredrickson, G. H.; Chmelka, B. F.; Stucky, G. D.; Science 1998, 279, 548.

7. Gu, C.; Chia, P. A.; Zhao, X. S.; Appl. Surf. Sci. 2004, 237, 387; Laha, S. C.; Mukherjee, P.; Sainkar, S. R.; Kumar, R.; J. Catal. 2002, 207, 213; Dai, Q.; Wang, X.; Chen, G.; Zheng, Y.; Lu, G.; Microporous Mesoporous Mater. 2007, 100, 268.

8. Zhang, Y.; Gao, F.; Wan, H.; Wu, C.; Kong, Y.; Wu, X.; Zhao, B.; Dong, L.; Chen, Y.; Microporous Mesoporous Mater. 2008, 113, 393.

9. Luz Jr., G. E.; Quintella, S. A.; Araujo, A. S.; Fernandes Jr., V. J.; Resumos do VII Encontro Norte, Nordeste e Centro-Oeste de Catálise, Salvador, Brasil, 2008.

10. Coutinho, A. C. S. L. S.; Quintella, S. A.; Araujo, A. S.; Barros, J. M.; Pedrosa, A. M. G.; Fernandes Jr., V. J.; Souza, M. J. B.; J. Therm. Anal. Calorim. 2007, 87, 457.

11. Araujo, A. S.; Jaroniec, M.; J. Colloid Interface Sci. 1999, $218,462$. 


\section{DESIDRATAÇÃO DE ETANOL SOBRE MATERIAL NANOESTRUTURADO DO TIPO LaSBA-15}

Geraldo E. Luz Jr.*

Coordenação de Química, Centro de Ciências da Natureza, Universidade Estadual do Piauí, Campus Poeta Torquato Neto, 64002-150 Teresina - PI, Brasil

Ana C. R. Melo, Stevie H. Lima, Antônio S. Araujo e Valter J. Fernandes Jr.

Departamento de Química, Universidade Federal do Rio Grande do Norte, 59072-970 Natal - RN, Brasil

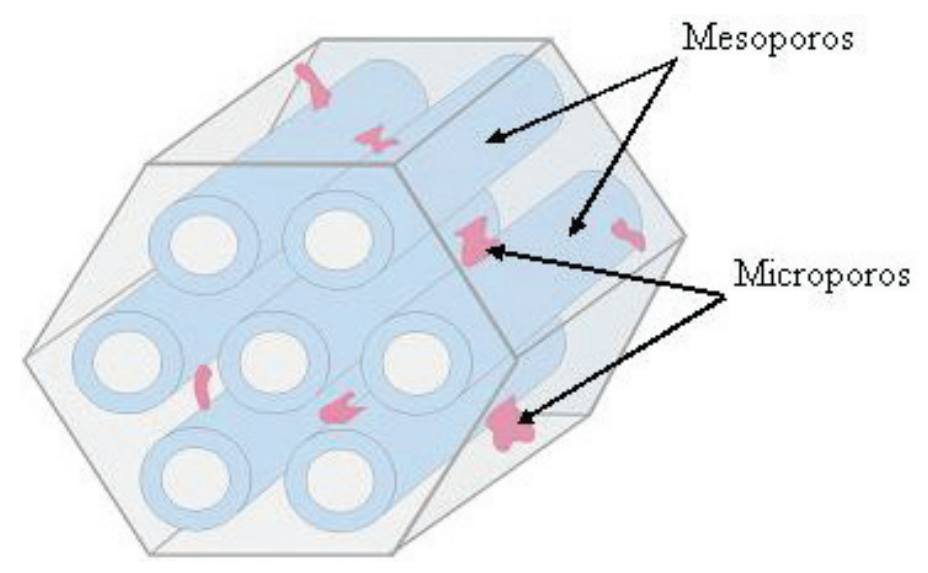

Figura 1S. Estrutura de poros da SBA-15 